\title{
Distributed Analysis: The Last Frontier?
}

\author{
Jorge Audy \\ Pontificia Universidade \\ Catolica, Porto Alegre, \\ Brazil \\ audy@pucrs.br
}

\begin{abstract}
$^{1}$
It is widely agreed that the trend toward distributed software development is growing. Although there are difficulties involved, this trend is here to stay, as organizations will continue to search for ways to develop software at lower cost but with same quality. Current research has focused primarily on the later stages of the software development life cycle, especially coding of software requirements. However, as organizations become more virtual, distributed development will become more apparent throughout the entire life cycle. In this study, we investigate distributed analysis in software development.

We report on results from a field experiment with two graduate level Information Systems classes, one located in Porto Alegre, Brazil and one in Chicago, U.S. The students in Brazil played the role of users whereas the students in Chicago role-played analysts. The Chicago-based students developed a requirements document for an information system by interviewing the Brazil students using an electronic discussion board. Our findings provide insight into the distributed analysis process and identify sources of potential problems.
\end{abstract}

\section{Introduction}

It is widely agreed that the trend toward distributed software development is growing [11]. Outsourcing of software development has become more common as organizations search for increased efficiency and lower costs. In particular, offshore outsourcing is attractive to organizations trying to take advantage of differential labor costs in other countries [1]. Although there are difficulties involved as well, this trend is here to stay, as

\footnotetext{
${ }^{1}$ Professors Evaristo and Watson-Manheim gratefully acknowledge support from the Center for Research in Information Management at the University of Illinois, Chicago, in conducting this research.
}

organizations will continue to search for ways to develop software at lower cost but with same quality.

At the same time, virtuality is increasingly common in organizations; more and more employees in different geographic locations are collaborating electronically. From a software development perspective, this means that users and stakeholders located in variety of different geographic locations, separated from each other and separated from systems analysts. Increasingly the trend toward distributed development will become more apparent throughout the entire life cycle, including analysis of software requirements in the early stages of the development life cycle.

Current research on distributed development has focused primarily on the latter stages of the life cycle, especially coding of requirements (e.g., [8]). We believe our study is one of the first to address distributed analysis in software development. Our goal is to understand the distributed analysis process better and to identify sources of problems. In addition, we take a first step in the development of training for software developers to increase effectiveness in the distributed environment.

In this study, we report on results from a field experiment with two graduate level Information Systems classes, one located in Porto Alegre, Brazil and one in Chicago, U.S. The students in Brazil played the role of users whereas the students in Chicago role-played analysts. The Chicago-based students developed a requirements document for an information system by interviewing the Brazil students using an electronic discussion board.

The paper is organized as follows: First, we review several different literature streams to establish the foundation for our research. Next we present the methodology used for our study. Finally we present the results and discuss the implication of the study for future research. 


\section{Background}

In the following sections, we first explore the role of requirements determination in the analysis process. The prevailing assumption in the literature has been that requirements determination usually takes place in a face-to-face (FTF) setting. As there has been little research on distributed analysis, we turn to literature from distributed collaboration and cross-cultural relations to develop a foundation for our research.

\subsection{Role of Requirements Determination in Distributed Analysis}

Requirements determination is a critical part of the software development process, occurring early in the life cycle. Correct and complete requirements lead to a more efficient development process and increased quality and acceptance of the completed software product [4]. Brown \& Rogich discuss requirements determination as a three-step process: 1) information gathering - eliciting requirements from users; 2) representation - modeling the elicited requirements in some physical fashion; and 3) verification - verifying with the user that the model of requirements is correct. We focus our study on the first step in the process, the initial elicitation of information from the user by the analyst.

Requirements determination, and especially information gathering, has long been an problematic area of software development $[4,6,15]$. Problems stem from a variety of sources, including human limitations, communication skills, and the complexity of the requirements determination task. Davis [6] discusses the limitations of humans in specifying information requirements. Limited short-term memory, bias toward more recent or available information and the need to simplify complex information are some factors that contribute to incomplete user requirements.

In addition the requirement definition task has "high dynamic complexity" [3]. This complexity stems from the evolutionary nature of the requirements, which are clarified only through multiple iterations of information gathering. Developing requirements is also dependent on input from a variety of different people using "same words to express very different concepts" [3]. The requirements task involves surfacing these differences where possible, and negotiating some consensus. Differences may not only be due to differences in interpretation and understanding [15] but also to differences in "vested interests" of the participants [3] further increasing the complexity of the task.

Having a structure to think about a problem helps reduce the inherent complexity [3]. The development of a methodology for requirements elicitation provides such a structure. In a recent study, Brown \& Rogich [4] propose a task-level model of the requirements elicitation task. The model addresses both cognitive and communication problems in the requirements elicitation task. The model proposes that the user has an understanding, implicit and explicit, of the problem space that the information system will address. The analyst, through dialogue with the user, must develop understanding of the problem space. Using this information and information from other sources (e.g., documents and company policies), the analyst develops the representation of the requirements, i.e., the requirements document.

Communications skills are then crucial to effective requirements elicitation, and the development of a common understanding of the problem is a key objective. Furthermore, ineffective communication in this process is negatively correlated with project success [15]. Although a number of other techniques have been proposed, interviews are the most commonly used requirements elicitation strategy $[4,7]$. The assumption is that elicitation of user requirements will largely be based on synchronous FTF communication between the user and the analyst. In their popular textbook, Dennis \& Wixom urge the analyst to establish rapport with the interviewee "so that he or she trusts you and is willing to tell you the whole truth (p.117)." They provide tips on developing interpersonal skills important for interviewing, including a recommendation to "watch body language (yours and theirs)" (p. 118).

Explicit strategies and tactics for effective communication between the analyst and stakeholders have generally not been addressed [15]. Beyond a discussion of interviewing techniques, communication skills training is largely ignored, there seems to be an implicit assumption that naturally occurring relationship development activities will take place in a FTF environment. In the distributed environment, however, FTF communication is often not feasible, and even synchronous electronic communication may become difficult.

Herbsleb and Mockus [9] find evidence that software development work conducted across distributed sites takes longer to perform than similar work at collocated sites in part due to the change in patterns of communication, leading to reduced understanding of background information and context 
at distant sites. While this study does not focus specifically on the requirements elicitation task, the results provide evidence that distributed communication can have a negative effect on the development process.

The lack of explicit training and reliance on implicit strategies occurring in FTF interactions leaves requirements elicitation particularly vulnerable to negative consequences of distributing the development process. There is need for explicit training strategies for the elicitation task. As there has been little research on this question in the current literature on software development, we next turn to recent literature on communication-related consequences of distributed work.

\subsection{Distributed Analysis as a Problem of Shared Understanding}

Recent research has begun to address the difficulties in communication in a distributed environment from a mutual knowledge perspective [5] or similarly, a shared mental model perspective [12]. Mutual knowledge is "knowledge that the communicating parties share in common and know they share" [5]. Hinds [12] discusses the concept of "perspective taking"- a process whereby people interact with the goal of understanding the others' view - in developing a shared mental model. Both authors point out communication problems that occur as a consequence of working in a distributed environment and have significant effect on the development of shared understanding between communication partners. This view is especially relevant when examining distributed software analysis where the goal of the dialogue between analyst and user is to develop a shared understanding of the focal problem space. We next review some of the problems faced by dispersed collaborators.

Cramton [5] finds geographically dispersed teams face significant problems maintaining mutual knowledge in collaborative endeavors. Dispersed team members in her study often did not discern which "features of their context and situation differed from the contexts and situation of remote partners and they did not communicate local information." While important to work across these differences to collaborate, her research suggests, "dispersed collaborators were not skilled at discovering and communicating such differences, and when differences were mentioned, remote partners sometimes failed to note or remember it."

In addition, the lack of shared context and mutual knowledge led to different interpretations of information. This had implications for development of trust, as problems were attributed to individuals rather than to situational misunderstandings. Cramton [5] points out also that communicating and collaborating in a distributed environment is complicated by the difficulties in investigating and integrating information from multiple locations. For example, how does one interpret silence from a communication partner? Is it simply due to feedback lag, is the receiver having difficulty understanding information, is the receiver "slacking off"? Multiple interpretations must be weighed increasingly the complexity of the communication.

The heightened uncertainty of the computermediated environment increases the importance of trusting behaviors in effective communication [14]. From the example above, a simple action such as timely message response may be interpreted as an indication of trust and increasing involvement between the communicators. The level of trust between communicators is important to developing the open dialogue important for software requirements elicitation. Jarvenpaa and Leidner [14] found that teams high on trust were able to solve problems and resolve conflicts in a distributed environment, where participants were limited to electronic communication.

Hinds [12] also investigates consequences of communication and collaboration in a non-collocated environment. She finds that distributed workers have less shared information, which remains unshared, and therefore develop different perspectives about the task. "Distributed workers have less overlap in their mental models of a task than do co-located workers, that context accounts for much of the discrepancy, and that distributed workers rarely discuss the contexts in which they are working.... Distributed teams may be less effective than co-located teams because they will be less able to understand their teammates' perspectives and will be less able to coordinate action." She discusses the need for ways to help team members "take teammates' perspective and develop a more shared image of the work."

Moreover, groups located in distant geographical locations are also likely to have different cultural make-ups. In this manuscript, we will adopt Hofstede's [13] cultural categorizations. Hofstede defined initially four cultural dimensions: power distance, individualism/collectivism, uncertainty avoidance, and femininity/masculinity. Later he added time horizon as a fifth dimension. The reader is referred to that source for a more detailed definition of the dimensions. In our case, the two sets of groups were over 5,000 miles apart and differed in culture. 


\subsection{Cross-Cultural Differences}

For purposes of this study, it is interesting to compare the dimensions where U.S. individuals are different from Brazilians. Table 1 presents a summary of the scores for the four cultural dimensions.

U.S. individuals are considerably more individualistic as compared to the Brazilians. Individuals with higher scores on individualism will tend to value their own advancement more than they are interested on the group's advancement. Brazilians have a higher score than U.S. individuals on power distance, suggesting that they are more accepting of differences in power between subordinates and managers. Brazilians also tend to value quality of life more than the U.S. individuals based on their femininity/masculinity score compared to U.S. Finally, Brazilians are less likely to be willing to deal with uncertainty than U.S. individuals. Brazilians also have a longer time horizon attitude than U.S. individuals.

A priori, the differences in cultural scores suggest a set of differences in expectations of the respective groups. The most important would be Brazilians displaying a combination of higher interest in quality of life - and relationships - coupled with a higher valuation of group objectives.

\section{Table 1: Summary of Cultural Scores [13]}

\begin{tabular}{|l|l|l|}
\hline & Brazil & U.S. \\
\hline Power Distance & 69 & 40 \\
\hline $\begin{array}{l}\text { Individualism / } \\
\text { Collect }\end{array}$ & 38 & 91 \\
\hline $\begin{array}{l}\text { Uncertainty } \\
\text { Avoidance }\end{array}$ & 76 & 46 \\
\hline $\begin{array}{l}\text { Masculinity } \\
\text { Femininity }\end{array}$ & 49 & 62 \\
\hline
\end{tabular}

\subsection{Problem statement}

A common theme in the research we have examined is that the lack of shared context inherent in a dispersed environment creates additional problems for developing shared understanding between communication partners. Cramton [5] points out that mutual knowledge is often referred to as "common ground" which is symbolic of the deeply engrained idea that mutual knowledge is dependent on co-presence. Similarly, Herbsleb et al. [10] find: "collaborations over distance must contend with the loss of the rich, subtle interactions that collocated teams use to coordinate their work."

Partly due to such difficulties, most training for eliciting user requirements has been developed under the assumption that the elicitation of software requirements by the analyst takes place most efficiently in the FTF environment. Our goal is to develop a better understanding of distributed analysis and to develop a training approach for improving context sharing - or "common ground" in Cramton's words - and consequently the elicitation of requirements in this environment.

\section{Methodology}

The research setting was a graduate level Information Systems and Organizations class at PUC/RS in Porto Alegre, Brazil, and a graduate level Systems Analysis and Design class at the University of Illinois, Chicago, taught by the first two authors, respectively. Students in each class were assigned to groups of 3-4 members. There were 3 groups in each class. The students in the SAD class were "analysts" who were to interview the "users" from Brazil. The assignment for the analysts was to understand the requirements for the public voting system in Brazil and to develop an interface for the voting system. The interviews and discussions between the students took place entirely through the electronic discussion feature of the Blackboard system (web-based course instructional site at UIC). Students were instructed not to use e-mail or communication media other than the discussion board. Students were given 30 days to complete the assignment.

All groups also completed a "lessons learned" document. Questions the students were to address in the "lessons learned" document included:

a. Your perception of effort of each team (US vs.

Brazil)

b. What worked best?

c. What did not work?

d. Adequacy of the medium (discussion threads) to the task

e. What were your concerns during the task?

f. What did you learn from this task?

In addition, the UIC students also turned in a description of the public voting system in Brazil. The students were informed that this project had dual objectives: 1) to give them experience in distributed analysis of software requirements and 2) the results 
would be used for research purposes. They were given the option of an alternative assignment, but no one chose this option.

\subsection{Training}

Selected groups from each class received training for collaboration in a non-collocated environment. This training had multiple objectives: 1) to raise awareness about miscommunication and lack of understanding in a distributed environment, 2) educate students about the underlying causes of this problem, in particular lack of mutual awareness of context, and 3) give students specific pointers for assessing how and when to share context. The training emphasized the importance of involvement of the communicator in understanding what contextual factors are important for the task being performed, e.g., time zone differences and expectations of task completion, and explicitly communicating this information. In addition, students were encouraged to help their communication partners to understand the importance of sharing context.

The instructor for each course trained randomly selected student groups. One Brazilian group was trained (Group A) and two US groups trained $(1,2)$. Groups of users (A, B, C) and analysts $(1,2,3)$ were paired. Groups at each university were not aware of which groups had been trained in the other university. Groups who were trained were asked not to discuss the training with groups who were not trained.

\section{Data Analysis}

In this section, we will present the data stemming from the reports submitted by the students on both universities. The U.S. reports, written from the perspective of a system analyst, had two sections: one, a description of the specifications for the electoral system in Brazil and the computerized interface; two, a "lessons learned" section. The Brazilian reports, written from the perspective of users, had only the lessons learned section. In this way, the report on lessons learned from group A, B and $C$ (Brazil) will be compared with the report from group 1, 2 and 3 (U.S.). These reports are seen as the "perceived" status of the exchange, whereas the messages exchanged between the respective group dyads represent the actual exchanges. A careful qualitative analysis of the differences between perceived and real exchanges will be performed based on the comparisons between the two "lessons learned" reports from the respective U.S. and Brazilian groups plus the complete collection of their written communication. At the end we will draw conclusions from this analysis.
In general, differences in language did not seem to be a problem for any of the groups, although there were clearly small hiccups here and there.

\subsection{Groups A (Brazil) / 1 (U.S.)}

Group 1 (score on the task of delivering specifications: 94) took to heart its training in context sharing. In fact, they were the only U.S. group that initiated the interaction. In their own words,

"When asking questions, we tried to be very specific and describe in detail every concept we felt might be vague for Brazilians. We did not automatically assume our peers know what we mean by the personal identifier, such as Social Security Number, or by the rating scale. Conversely, sometime we needed to ask for further explanation on what Brazilian students submitted (e.g.: "...he receives its heading from voter and the voucher from voting..."). The main difficulty encountered in the project was the different context of the time pressure. For instance, our Brazilian counterparts did not share our sense of urgency, awareness of the tight schedule and excessive workload. Specifically, our context could be described as: "We only have several weeks for this project; we need to manage this efficiently. This is a cool and interesting experience but there are too many other assignments and exams to prepare for. We can't afford to spend more time than necessary on this". Conversely, the context for Brazilian students seemed to be: "Whatever this is about, it is an exciting exercise. We need to use this opportunity to exchange information with American students, to learn from them and promote ourselves."”

Their perceptions of the process were supported by the analysis of their emails to group 1 in Brazil, the only group trained in Brazil. They initiated the interviewing process by sending a rapid succession of emails (April $1^{\text {st }}$ to April $4^{\text {th }}$ ) introducing everybody. In the next couple of days, the Brazilian group trickled in with introductory messages, member by member. In some cases, these were detailed messages including the URL for a personal home page. By April $7^{\text {th }}$, one of the members of the U.S. 
group sent a not-so-subtle message that they "meant business:"

"Hi! All, Thanks for such a nice introduction of $u$ guys. hope $u$ all had a nice weekend. As we are aware, we have not much time left lets get going with our ptoject. we all here in UIC are anxiously waiting for detailed information regarding the present voting system of Brazil and the requirement of the system. hope we get the required information very soon.take care."

After another few exchanges fairly personal in tone from the Brazilian group, the U.S. group reiterated, on April $8^{\text {th. }}$

"Alright guys, thank you very much for
very cordial response!!! Looks like we all got to know each other, at least virtually. Now that we successfully carried out the introductory and social part, let's move on to the project.

I'd suggest you brazilian guys start by introducing us to your voting system: anything you find important and interesting: physical interface (e.g. do you use touchscreen/keyboard?, is it interactive and easy to use even for the first-time user? is it time efficient, do you find the design of screens appealing? etc etc.) Each of you can submit your own experience and opinions, that would improve the objectivity. Then, I suggest, we will elaborate more on the parts of your report that will need further details."

This message was followed almost immediately by a flurry of general information about Brazil, economy of the state where the Brazilian University is located, and a few more generalities. As a result, the U.S. group sent a long list of specific questions, which met with a short silence. When another U.S. group member sent a further set of questions before the previous ones were answered, the Brazilian group responded with a somewhat annoyed short note:

"Guys, In the monday, we will be answering all its questions and sending bigger detailing of our politico system and as we make the election. You forgive the delay, and you have a little of patience. Thank you all very much and have a nice weekend."

And as soon as a couple of answers came through, the U.S. students tried to give some (somewhat misguided, as we will see below from the Brazilian students' reaction) positive reinforcement:

"Hello Team, it seems that information has started to flow. Better late than never.. Well I would like to mention one more thing that our team is lagging behind from other teams. This is what I felt after the discussion in the class yesterday."

Group 1 went further in their report to explain that they were not sure how much the assignment was worth on the other side, and explicitly avoided sharing how much the assignment was worth on the U.S. side to avoid having the Brazilians decrease their involvement.

Although the report from Group 1 suggests that everything went very well ("All of us agreed that the Brazilian team was extremely pleasant to work with"), an analysis of Group A's report showed a completely different picture. Group A thought that the "immense" questionnaire just steamrolled their planned organization of efforts in sharing information with the "analysts"; that the tool was not appropriate to the task at hand because of the lack of synchronicity, location of shared files in separate areas of the site than the actual threads; and too many clicks to access either area.

Most importantly, the Brazilians thought that their effort at providing what they considered to be context and background information was seem as irrelevant by the U.S. students - in fact, a waste of time which was a reason for complaints and insistent "prods." Their trust on their U.S. counterparts started very high, dropping when feedback was negative, and went up again at the end when the U.S. students showed high knowledge on the subject.

Very tellingly, the Brazilian students ended their report with the following comment:

"They [the U.S. students] seemed to be direct and objective in their efforts, were more concerned with the task than with the interaction itself. For us the interaction, communication exchange was the main focus of the activity (nontechnical aspects). ... In conclusion, it seems that the objectives of the two teams were not the same." 


\subsection{Groups B (Brazil) / 2 (U.S.)}

Group B (not trained) started the interaction with a short introduction. A small number of emails followed from both sides including a longish questionnaire sent by the U.S. students (Group 2), who had been trained in context sharing. In response, one Brazilian student emailed with a breakdown of which other Brazilian members would answer which question. Both groups liked using the discussion board, with the Brazilians longing for a richer media.

In Group 2's perspective (score on the task of delivering specifications: 87), the performance was good, and only had the risk of breaking down when the following happened, in the U.S. student's words:

"For example when we asked about the "Blank" key, asking it in a manner like, "No one really told us what the "Blank" key definitively performs. We require additional details about this key." would cause communication channels to actually deteriorate rather then improve them."

The Brazilian students, all with prior system analyst training, disagreed. They noticed that the U.S. students were only interested in specific details about the electoral system, not about a more general background to base their analysis; as a consequence, there was no structure to the questions or requirements analysis. This was particularly evident in the fact that U.S. students sent a questionnaire before the background and domain were established. In their opinion, the U.S. group also failed to give feedback related to their understanding of what had been transmitted. As a result, they had doubts whether the U.S. students truly understood the system.

Further light on the matter can be gleaned from the U.S. report, where students say "we first wanted to obtain a general idea of their voting system." This is in stark opposition to what the Brazilian students perceived as total lack of interest in the generals of the situation.

The Brazilian students also showed disappointment in the fact that the U.S. students were not interested in a more personal involvement. In fact, from a total of 21 messages exchanged, only 4 originated in Chicago. Another source of disappointment for the Brazilians was the fact that the U.S. students summarily dismissed the structure the Brazilians created. Moreover, the perceived level of "stress and coldness" in the U.S. messages clearly left a bad taste in the Brazilian's minds. As a consequence, the high starting level of trust tended to fall continuously.

\subsection{Groups C (Brazil) / 3 (U.S.)}

In this case, neither of the groups was trained in context sharing. The U.S. students (score on the task of delivering specifications: 95) seemed to enjoy using the discussion board, although pointing out the fact that threaded discussions were not ideal for data summarization, for instance. The Brazilian students were generally satisfied with the process, although unhappy with the following limitations. First, the U.S. students were too quick in sending out a list of questions, which somewhat threw the Brazilian students in a state of temporary confusion, since they had already planned to share certain pieces of background information. Second, most of the interaction was done with only one U.S. student, which limited the breadth of the interaction. On the other hand, this particular individual took the trouble to learn a few words in Portuguese and as a result was perceived as "committed" to relationship building. This may have affected their perceived trust level, which was very high during the whole period.

An interesting level of misunderstanding occurred when the Brazilians described the voting system as

"There is a small terminal (with some buttons and a screen) hardwired to the electronic ballot box to validate the voting card number and to qualify the beginning of the voting process. The electronic ballot box is a type of device (a small box) with a screen, buttons of numbers (0-9) and three keyboard keys (Confirm, Correct, White)."

Eventually it was clarified that "white" was a literal translation of the Portuguese word "branco" which in fact should have been translated as "blank", since it means a vote that has been intentionally cast without any candidate written on it.

\section{Data Discussion}

The main objective of this study was to better understand of distributed analysis as well as to start developing a training approach for context sharing. The initial training program was designed to cover the main problems that had been identified under a relatively limited set of circumstances. This experiment proved to be a challenge to our training plan, and has in fact generated powerful suggestions for improvements. In the next few paragraphs, we 
will discuss what the data presented in the previous section.

At a first glance, it seems that the only group that was trained in Brazil took context sharing to mean something different than what was understood as context sharing by their U.S. counterparts. Brazilians tended to "contextualize" their understanding of context based on their cultural reality, and assumed that sharing general and personal background would do the trick. This was, however, seen by the U.S. group as mostly irrelevant and only delaying their completion of the task.

We speculate that cultural differences would have played a strong role in these differences. Brazilians are more likely to be collectivistic (as opposed to individualism, which runs very high in the U.S. culture, to borrow Hofstede's terms) and as a result more likely to value highly warm personal relationships. Nothing could be farther from the U.S. students' intentions. In fact, this observation was present in other groups as well, regardless of training or not in context sharing: the U.S. emphasis on task, versus the Brazilian emphasis on relationships.

The experience of the other two groups $(\mathrm{B} / 2$ and $\mathrm{C} / 3$ ) was not as extreme. Group 2 was trained in context sharing. Their Brazilian counterparts were not very happy with their "relationship skills" and seriously doubted their technical knowledge. Finally, Group C/3 performed well and by and large bypassed all problems demonstrated by the other groups, even though neither had been trained in context sharing.

Based on the limited amount of data available in this study, the training program in context sharing did not seem to clearly affect performance in distributed analysis. We believe that there may be reasons for such outcome. First, the number of groups involved is too small to generalize results; groups $\mathrm{C} / 3$, for instance, may just have been the highest performing groups and intuitively may have done things prescribed in the context sharing training program (such as learning each other's words/metaphors/"language") even though they had not been trained for such. Therefore, it could be that group differences are still the best predictor of performance. But the question remains: for competence-matched groups, would training in context sharing improve performance?

The data also showed some commonalities across all three groups. Their respective cultural makeup seemed to be one of the strongest predictors of the expectations in task performance and proportional interest in relationship building instead. A closer look, however, shows that there were alternative explanations for such difference. The most likely is the difference in points devoted to the assignment: $5 \%$ of the total grade for U.S. students and $30 \%$ for Brazilian students. On a pure costeffectiveness basis, the U.S. students may not have been to willing to spend more than the smallest amount of time possible in the assignment - or to "squeak by." Moreover, such difference could also explain the reason two of the U.S. groups seemed to be too eager to jump into actual long questionnaires instead of first gathering a more general situation analysis, even though that issue had been discussed in the systems analysis and design class a few weeks earlier in the context of gathering requirements. This shortcut attitude was particularly noticed by the Brazilian students who all had very strong background in Systems Analysis and Design due to their undergraduate coursework and in some cases even work experience.

Naturally, it would be hard to eliminate the explanation of cultural differences as a potential partial cause for such differences in expectations. The learning from this fact is that the next experiment should devise ways to eliminate confounds, either by using same weights on grades on all participants or conversely by eliminating the cultural difference.

Finally, alternative explanations for the results could also originate from the fact that the three U.S. groups had different levels of competence or effort spent in the assignment, something that was evident from the grade assigned by the professor to their respective reports.

\section{Conclusion}

We set out to improve our understanding of distributed analysis with emphasis on the role of context sharing. We stopped short of conclusively showing that such training has a positive or negative influence on the quality of distributed analysis; however, we learned a lot about some of the issues associated with distributed analysis, particularly in a cross-cultural environment. Expectations about the role of task versus relationships may be one of the critical cultural differences - fortunately, something that can be at least brought into the open fairly easily by surfacing group assumptions before the work starts.

The fact that we had a very small sample of noncompetence-matched student groups could have been the critical reason for lack of more conclusive results. However, we learned that context sharing can (and will) be perceived differently based on one's cultural context; that further studies should be more carefully matched on both group performance and on grade weights (the Brazilian students derived 30\% of their final grade from their participation and reports, whereas the U.S. students only got $5 \%$ of their final grade the same way). The impact of culture on 
context sharing needs to be revised and appropriately dealt with as well. In addition to fixing such shortcomings, further studies should adapt the context sharing training based on current findings.

\section{References}

[1]R. Agarwal, Global Outsourcing for IT, in SIM Webcast, April 29. 2003, SIM:

[2]D. Arnold and F. Niederman, "The Global Workforce," Communications of the ACM, vol. 44, no. 7, 2001, pp. 31-33.

[3]R.O. Briggs and P. Gruenbacher, "Easy WinWin: Managing Complexity in Requirements Negotiation with GSS," in Proceedings of 35th Annual Hawaii International Conference on Systems Science, Hawaii, 2002, IEEE.

[4]G.J. Brown and M.B. Rogich, "An Empirical Investigation of User Requirements Elicitation: Comparing the Effectiveness of Prompting Techniques," Journal of Management Information Systems, vol. 17, no. 4, 2001, pp. 223-249.

[5]C.D. Cramton, "The Mutual Knowledge Problem and its Consequences for Dispersed Collaboration," Organization Science, vol. 12, no. 3, 2001, pp. 346371 .

[6]G. Davis, "Strategies for Information Requirements Determination," IBM Systems Journal, vol. 21, no. 1, 1982, pp. 4-30.

[7]A. Dennis and B. Wixom, Systems Analysis and Design, ed. Beth Golub, New York: John Wiley and Sons, 2000.

[8]R. Grinter, J. Herbsleb, and D. Perry, "The Geography of Coordination: Dealing with Distance in R\&D Work," in Proceedings of International ACM SIGGROUP Conference on Supporting Group Work, Phoenix, AZ, 1999, ACM Press.

[9]J. Herbsleb and A. Mockus, "An Empirical Study of Speed and Communication in Globally Distributed Software Development," IEEE Transactions on Software Engineering, vol. 29, no. 6, 2003, pp. 481494.

[10]J. Herbsleb, A. Mockus, T. Finholt, and R. Grinter, "Distances, Dependencies and Delay in a Global Collaboration," in Proceedings of ACM Conference on Computer Supported Cooperative Work, Philadelphia, PA, 2000, ACM Press.
[11]J. Herbsleb, A. Mockus, T. Finholt, and R. Grinter, "An Empirical Study of Global Software Development: Distance and Speed," in Proceedings of 23rd International Conference on Software Engineering, 2001.

[12]P. Hinds, Perspective taking among distributed workers: The effect of distance on shared mental models, Working Paper \# 6, Stanford University, 2000.

[13]G. Hofstede, Culture Consequences, Newbury Park, California: Sage Publications, 1984.

[14]S. Jarvenpaa and D.E. Leidner, "Communication and Trust in Global Virtual Teams," Organization Science, vol. 10, no. 6, 1999, pp. 791-815.

[15]C. Urquhart, "Themes in early requirements gathering: The case of the analyst, the client, and the student assistance scheme," Information, Technology and People, vol. 12, no. 1, 1999, pp. 44-70. 